${ }^{1}$ Department for Theoretical Physics, Ivan Franko National University of Lviv (12, Dragomanov Str., 79005 Lviv, Ukraine)

2 College of Natural Sciences, Ivan Franko National University of Lviv (107, Tarnavs'kyi Str., Lviv, Ukraine; e-mail: gpanochko@gmail.com)

\title{
THE EFFECTIVE MASS OF AN IMPURITY ATOM IN THE BOSE LIQUID WITH A DEFORMED PACS 05.30.Jp, 02.40.Gh HEISENBERG ALGEBRA
}

\begin{abstract}
We consider the movement of $a^{3}$ He impurity atom in the Bose liquid. We suggest to describe the many-particle correlations between atoms of the Bose liquid, by using a deformed Heisenberg algebra. As generalized coordinates, we choose the collective variables that are the Fourier components of fluctuations of the density of Bose particles. The wave function of the investigated system in the zeroth approximation is the product of the wave function of the liquid helium-4 within deformed commutation relations between generalized coordinates and momenta and the plane wave of the impurity atom. We calculate the correction to the groundstate energy of the system "Bose liquid plus impurity" and the effective mass of the impurity atom ${ }^{3} \mathrm{He}$, by assuming that the boson-impurity interaction is a small perturbation.

Ke ywords: boson systems, deformed Heisenberg algebra, effective mass.
\end{abstract}

\section{Introduction}

The investigation of the impurity states of Bose and Fermi systems is intensified in connection with the consideration of spin-polarized hydrogen, hightemperature superconductors, and the experimental discovery of a Bose condensate by cooling the diluted gases of alkali metals. The study of the behavior of impurities in mixtures ${ }^{3} \mathrm{He}^{-}{ }^{4} \mathrm{He}$ and in superfluid ${ }^{4} \mathrm{He}$ is also relevant. Here, the important issue is the description of quantum states of the system "the Bose liquid plus the impurity atom", by modeling the interaction potential between atoms of the Bose liquid and the impurity atom. The consideration of manyparticle correlations between atoms in this system will give the qualitative and quantitative estimation of the effective mass of impurities.

Well promising are the studies of the dynamics of strongly related impurities in the one-dimensional Bose liquid [1]. With the development of various methods of cooling of Bose gases for the observation of the Bose condensation phenomenon, the interest in the studies of the tunneling effect for Bose particles in traps with strong boson-impurity interaction has increased [2]. The depletion phe-

(C) I.O. VAKARCHUK, G. PANOCHKO, 2017

ISSN 2071-0194. Ukr. J. Phys. 2017. Vol. 62, No. 2 nomenon of the Bose condensate by moving impurities $\left(\mathrm{Na}^{+}, \mathrm{Rb}^{+}, \mathrm{Yb}^{+}\right)$was examined in the recent experimental work [3]. The presence of impurity atoms in the Bose condensate leads to many exciting phenomena. An example is the creation of a two-electron bound state, which is called a bipolaron. It was found that they participate in atypical pairing mechanisms of high-temperature superconductivity.

A lot of papers are devoted to the investigation of impurity states and the calculation of the effective mass of impurity atoms in the Bose systems, in particular, within the Feynman variational formalism [4]. If the Bose condensate is described by the Fröhlich Hamiltonian, Feynman's variational approach is used for the calculation of the impurity effective mass. In work [5], a class of variational Gauss wave functions was proposed for the description of Fröhlich polarons at finite momenta. The effective masses of polarons of ${ }^{41} \mathrm{~K}$ and ${ }^{133} \mathrm{Cs}$ impurities in the Bose-Einstein condensate (BEC) of ${ }^{87} \mathrm{Rb}$ atoms, as well as the effective mass of ${ }^{6} \mathrm{Li}$ in $\mathrm{BEC}$ of ${ }^{23} \mathrm{Na}$ atoms, were calculated in [5]. The self-energy as a function of the scattering length parameter in the third order of perturbation theory with the use of ladder diagrams and the effective mass of the impurity were obtained in work 
[6]. In work [7], it was shown that, in the long-wave limit, the strong interaction between Bose particles of a two-component ultracold Bose gas with nonzero spins led to an increase of the value of effective mass of particles. In work [8], the lifetime of deuterium in a chamber, whose walls are covered with liquid saturated ${ }^{4} \mathrm{He}$, was considered, and the possibility of assessment of the effective mass of the impurity was discussed. In work [9], the properties of an impurity immersed in a dilute Bose gas at the zero temperature were investigated by quantum Monte-Carlo methods.

The analytical and numerical results for the effective mass of impurity ${ }^{3} \mathrm{He}$ at $T \rightarrow 0 \mathrm{~K}$ in a Bose liquid were obtained for the first time in the work by Slusarev and Strzemechnyj [10] in the frame of the Brillouin-Wigner perturbation theory $\left(M^{*} / M=\right.$ $=2.4$ ). In work [11], the wave function in the zeroth approximation for the system "Bose liquid plus the impurity atom" was chosen in the form of a product of the wave function of the ground state of the Bose liquid and the wave function of an isolated atom ${ }^{3} \mathrm{He}$. Having formulated the perturbation theory on such states, the authors obtained the numerical value of effective mass $M^{*} / M=1.81$ with regard for the structure factor calculated in [12]. The value $M^{*} / M=1.7$ was obtained by the variational method. In work [14], where the interaction between atoms ${ }^{4} \mathrm{He}$ and an impurity atom ${ }^{3} \mathrm{He}$ was modeled by the Aziz potential, and the value $M^{*} / M=2.15$ with the use of the experimental structure factor [15] was obtained in the second order of perturbation theory. The study of the low-temperature behavior of the effective mass of an impurity [16] was based on the averaging of the full statistical operator of a system "Bose liquid plus impurity" over the states of pure ${ }^{4} \mathrm{He}$. We also note that, in work [17], the chemical potential, immersion energy, and effective mass of ${ }^{3} \mathrm{He}$ in a Bose liquid were obtained by the Monte-Carlo method.

The growth of the effective mass of impurity ${ }^{3} \mathrm{He}$ in superfluid ${ }^{4} \mathrm{He}$ can be explained by the existence of the bound states of ${ }^{3} \mathrm{He}$ and ${ }^{4} \mathrm{He}$ in liquid and in pure ${ }^{4} \mathrm{He}$ due to the appearance of Cooper pairs [18]. This fact is confirmed by the experimental data on the effective mass of the impurity ${ }^{3} \mathrm{He}, M^{*} / M=$ $=2.15$, in the mixture ${ }^{4} \mathrm{He}-{ }^{3} \mathrm{He}$, when $T \rightarrow 0$ and the concentration of impurity atoms is small [19].

In our work, we will investigate the impurity states of the system "liquid ${ }^{4} \mathrm{He}$ plus one impurity atom
${ }^{3} \mathrm{He}$ " in the low-temperature limit. We propose an approach, which allows us to consider the nonlinear fluctuations between atoms of the liquid by introducing a deformed Heisenberg algebra with the generalized coordinates and momentum. The deformed Heisenberg algebra was first proposed in [21]. For this algebra, we choose generalized coordinates and the corresponding generalized momenta, so that the commutator between these variables is a quadratic function of the generalized coordinates with some deformed parameter [22]. We assume that this deformed function contains all anharmonic terms generated by the strong interaction between Bose particles. We develop a perturbation theory, by assuming that the interaction between the impurity and atoms of the Bose liquid is small. We calculate corrections to the ground-state energy of the system, by summing over the wave vector. We discuss the problem of the effective mass of the impurity ${ }^{3} \mathrm{He}$ in liquid ${ }^{4} \mathrm{He}$ at $T \rightarrow 0$. We remark that the deformation parameter in our calculations is fixed. Note that the wave function of the ground state of the system "Bose liquid plus of the impurity atom" in this approach is applicable not only to the calculation of the effective mass, but also for the evaluation of the immersion energy of the impurity.

\section{Statement of the Problem}

We consider a collection of spinless Bose-particles of mass $m$ and with Cartesian coordinates $\mathbf{r}_{1}, \ldots, \mathbf{r}_{N}$, which are situated in a volume $V$ together with an impurity atom of mass $M$ and a coordinate r. For the study of the impurity states of the many-boson system, it is convenient to use the representation of collective variables:

$\rho_{\mathbf{k}}=\frac{1}{\sqrt{N}} \sum_{j=1}^{N} e^{-i \mathbf{k} \mathbf{r}_{j}}, \quad \rho_{\mathbf{k}}=\rho_{\mathbf{k}}^{c}-i \rho_{\mathbf{k}}^{s}, \quad \mathbf{k} \neq 0$,

which are the Fourier components of the density fluctuations of Bose particles. The Hamiltonian of the system "Bose liquid plus impurity" can be represented as a sum

$\widehat{H}=\widehat{H}_{I}+\widehat{H}_{L}+\widehat{H}_{\text {int }}$.

Here, $\widehat{H}_{I}$ is the Hamiltonian of the impurity, being its operator of kinetic energy:

$\hat{H}_{I}=-\frac{\hbar^{2} \nabla^{2}}{2 M}$,

ISSN 2071-0194. Ukr. J. Phys. 2017. Vol. 62, No. 2 
here $-i \hbar \nabla$ is the operator of momentum of the impurity atom.

The second term $\widehat{H}_{L}$ is the Hamiltonian of the Bose liquid, which can be written in the $\rho_{\mathbf{k}}$-representation as

$\widehat{H}_{L}=\sum_{\mathbf{k} \neq 0} \frac{\hbar^{2} k^{2}}{2 m}\left(-\frac{\partial^{2}}{\partial \rho_{\mathbf{k}} \partial \rho_{-\mathbf{k}}}+\frac{1}{4} \rho_{\mathbf{k}} \rho_{-\mathbf{k}}-\frac{1}{2}\right)+$
$+\frac{N(N-1)}{2 V} \nu_{0}+\frac{N}{2 V} \sum_{\mathbf{k} \neq 0} \nu_{k}\left(\rho_{\mathbf{k}} \rho_{-\mathbf{k}}-1\right)+\Delta \widehat{H}$,

where $\Delta \widehat{H}$ contains all anharmonic terms associated with fluctuations of the particle density:

$$
\begin{aligned}
& \Delta \widehat{H}=\sum_{\substack{\mathbf{k} \neq 0 \\
\mathbf{k}+\mathbf{k}^{\prime} \neq 0}} \sum_{\mathbf{k}^{\prime} \neq 0} \frac{\hbar^{2}\left(\mathbf{k k}^{\prime}\right)}{2 m \sqrt{N}} \rho_{\mathbf{k}+\mathbf{k}^{\prime}} \frac{\partial^{2}}{\partial \rho_{\mathbf{k}} \partial \rho_{\mathbf{k}^{\prime}}}+ \\
& +\sum_{n \geq 3} \frac{(-)^{n}}{4 n(n-1)(\sqrt{N})^{n-2}} \times \\
& \times \sum_{\substack{\mathbf{k}_{1} \neq 0 \\
\mathbf{k}_{1}+\ldots+\mathbf{k}_{n}=0}} \ldots \sum_{\substack{\mathbf{k}_{n} \neq 0\\
}} \frac{\hbar^{2}}{2 m}\left(k_{1}^{2}+\ldots+k_{n}^{2}\right) \rho_{\mathbf{k}_{1} \ldots} \rho_{\mathbf{k}_{n}},
\end{aligned}
$$

and $\nu_{k}$ is the Fourier image of the interaction potential of Bose particles:

$\nu_{k}=\int \Phi(R) e^{i \mathbf{k} \mathbf{R}} \mathrm{d} \mathbf{R}$.

The last term in relation (2) $\widehat{H}_{\text {int }}$ is the operator, which involves the interaction of atoms of the liquid with the impurity atom:

$\hat{H}_{\mathrm{int}}=\frac{N}{V} \bar{\nu}_{0}+\frac{\sqrt{N}}{V} \sum_{\mathbf{k}_{1} \neq 0} \bar{\nu}_{k_{1}} \rho_{\mathbf{k}_{1}} e^{i \mathbf{k}_{1} \mathbf{r}}$.

Here, $\overline{\nu_{k}}$ is the Fourier image of the interaction potential of the system "impurity plus Bose liquid".

A specific feature in the studies of the quantum states of the system of Bose particles with Hamiltonian (4) is the correct consideration of the contribution $\Delta \hat{H}$. Now, we suppose that the canonical collective variables $\rho_{\mathbf{k}}$ and canonically conjugate momenta $-i \hbar \partial / \partial \rho_{\mathbf{k}, \mu}$ are replaced by some generalized coordinates $\widehat{Q}_{\mathbf{k}, \mu}$ and momenta $\widehat{P}_{\mathbf{k}, \mu}$ which satisfy the deformed commutation relation

$\left[\widehat{Q}_{\mathbf{k}, \mu}, \widehat{P}_{\mathbf{k}, \mu}\right]=i \hbar\left(1+\beta_{k} \widehat{Q}_{\mathbf{k}, \mu}^{2}\right)$.
The replacement we have made allows us to take the operator $\hat{\Delta} H$ into account in some effective manner. Then the Bose liquid can be interpreted as a collection of noninteracting harmonic oscillators with constant frequencies and a contribution from the anharmonicity of oscillations we propose to describe by the deformed Heisenberg algebra (8) with deformation parameter $\beta_{k}$.

Our task is to estimate the effective mass of the impurity. We will calculate corrections to the groundstate energy of the system "Bose liquid plus impurity," by using stationary perturbation theory. We assume that the operator $\widehat{H}_{\text {int }}$ is a small perturbation.

\section{Quantum States}

\section{of the System "Bose Liquid Plus} Impurity" with the Deformed Algebra

We suppose that the wave function of the system "Bose liquid plus impurity" can be written as a product of the wave function of the Bose liquid $|\mathbf{k}\rangle$ and the wave function of the impurity atom:

$|\mathbf{k}, \mathbf{q}\rangle=\frac{1}{\sqrt{V}} e^{i \mathbf{q r}}|\mathbf{k}\rangle$.

To obtain solutions of the Schrödinger equation (4) for the Bose liquid with the deformed commutation relation (8), we use the standard transformation from the operators $\widehat{Q}_{\mathbf{k}, \mu}$ and $\widehat{P}_{\mathbf{k}, \mu}$ to a new pair of canonically conjugate variables $q_{\mathbf{k}, \mu}$ and $p_{\mathbf{k}, \mu}$ :

$\beta_{k}>0$ :

$\widehat{P}_{\mathbf{k}, \mu}=\widehat{p}_{\mathbf{k}, \mu}, \quad \widehat{Q}_{\mathbf{k}, \mu}=\frac{\tan \left(\widehat{q}_{\mathbf{k}, \mu} \sqrt{\beta_{k}}\right)}{\sqrt{\beta_{k}}}$,

$\beta_{k}<0$ :

$\widehat{P}_{\mathbf{k}, \mu}=\widehat{p}_{\mathbf{k}, \mu}, \quad \widehat{Q}_{\mathbf{k}, \mu}=\frac{\tanh \left(\widehat{q}_{\mathbf{k}, \mu} \sqrt{\left|\beta_{k}\right|}\right)}{\sqrt{\left|\beta_{k}\right|}}$,

where

$\left[\widehat{q}_{\mathbf{k}, \mu}, \widehat{p}_{\mathbf{k}, \mu}\right]=i \hbar$.

We can also conclude that the wave function of the Bose liquid depends on the sign of the deformation parameter $\beta_{k}$.

The wave functions of the Bose liquid for (10) and (11) can be written as follows:

$|\mathbf{k}\rangle=\prod_{\mathbf{k} \neq 0}^{\prime} \prod_{\mu=c, s} \psi_{n_{\mathbf{k}, \mu}}\left(q_{\mathbf{k}, \mu}\right)$. 
Here, the quantum numbers $n_{\mathbf{k}, \mu}=0,1,2, \ldots$, and $\mu=c, s$.

The detailed analysis of the solutions of the Schrödinger equation with Hamiltonian (4) for (10) and (11) showed that the deformation parameter $\beta_{k}$ should be chosen negative for liquid helium [22].

We write the explicit form of the wave function for a fixed mode in (13), when $\beta_{k}<0$ :

$$
\begin{aligned}
& \psi_{n}\left(q_{\mathbf{k}, \mu}\right)=\left|\beta_{k}\right|^{1 / 4} \sqrt{\frac{\Gamma(\nu-n+1 / 2) \Gamma(2 \nu-2 n+1)}{n ! \Gamma(1 / 2) \Gamma(\nu-n) \Gamma(2 \nu-n+1)}} \times \\
& \times\left(-\frac{d}{d x}+\nu \tanh x\right) \ldots\left(-\frac{d}{d x}+(\nu-n+1) \tanh x\right) \times \\
& \times \cosh ^{-\nu+n} x .
\end{aligned}
$$

Here,

$x \equiv q_{\mathbf{k}, \mu} \sqrt{\left|\beta_{k}\right|}, \quad n \equiv n_{\mathbf{k}, \mu}$.

In particular, the ground-state wave function of the Bose liquid (when all quantum numbers $n_{\mathbf{k}, \mu}$ are equal to zero) for $\beta_{k}<0$ reads

$$
\begin{aligned}
& |0\rangle=\prod_{\mathbf{k} \neq 0}^{\prime} \prod_{\mu=c, s}\left|\beta_{k}\right|^{1 / 4} \sqrt{\frac{\Gamma(\nu+1 / 2)}{\Gamma(1 / 2) \Gamma(\nu)}} \times \\
& \times \cosh ^{-\nu}\left(q_{\mathbf{k}, \mu} \sqrt{\beta_{k}}\right) .
\end{aligned}
$$

Here,

$\nu=-\frac{1}{2}+\frac{\alpha_{k}}{\left|\beta_{k}\right|} \sqrt{1+\left(\frac{\left|\beta_{k}\right|}{2 \alpha_{k}}\right)^{2}}$,

where $\alpha_{k}$ is the Bogoliubov factor:

$\alpha_{k}=\sqrt{1+\frac{2 N}{V} \nu_{k} / \frac{\hbar^{2} k^{2}}{2 m}}$.

Let us write the wave function of the first excited level of the system "Bose liquid plus impurity" for the $\mathbf{k}^{\prime}, \mu^{\prime}$ mode and the wave vector of the impurity $\mathbf{q}^{\prime}$. We suppose that, in the set of quantum numbers $n_{\mathbf{k}, \mu}$, the number $n_{\mathbf{k}^{\prime}, \mu^{\prime}}=1\left(\right.$ when $\left.\mathbf{k}^{\prime}=\mathbf{k}, \mu^{\prime}=\mu\right)$, and other quantum numbers $n_{\mathbf{k}, \mu}=0$ (when $\mathbf{k}^{\prime} \neq \mathbf{k}$, $\left.\mu^{\prime} \neq \mu\right)$. Then

$\left|\mathbf{k}^{\prime}, \mathbf{q}^{\prime}\right\rangle=\frac{1}{\sqrt{V}} e^{-i \mathbf{q}^{\prime} \mathbf{r}}\left|\mathbf{k}^{\prime}\right\rangle$

Here, $\left|\mathbf{k}^{\prime}\right\rangle$ is the wave function of the excited state of the Bose liquid for $\beta_{k}<0$ :

$\left|\mathbf{k}^{\prime}\right\rangle=\sqrt{2(\nu-1)} \sinh \left(q_{\mathbf{k}^{\prime}, \mu^{\prime}} \sqrt{\left|\beta_{k^{\prime}}\right|}\right)|0\rangle$.
In the limit $\beta_{k} \rightarrow 0$, the wave function of the system "Bose liquid plus impurity" in the first excited state is:

$$
\begin{aligned}
& \left|\mathbf{k}^{\prime}, \mathbf{q}^{\prime}\right\rangle=\frac{1}{\sqrt{V}} e^{-i \mathbf{q}^{\prime} \mathbf{r}} \sqrt{2 \alpha_{k^{\prime}}} \rho_{-\mathbf{k}^{\prime}, \mu^{\prime}}|0\rangle, \\
& |0\rangle=\prod_{\mathbf{k} \neq 0}^{\prime} \sqrt{\frac{\alpha_{k}}{2 \pi}} e^{-\frac{1}{2} \alpha_{k} \rho_{\mathbf{k}} \rho_{-\mathbf{k}}} .
\end{aligned}
$$

\section{Corrections to the Ground-State Energy of the System "Bose Liquid Plus Impurity" with a Deformed Heisenberg Algebra}

Let as suppose that the Bose liquid is in the ground state with the wave function given by Eq. (14). The ground-state energy

$$
\begin{aligned}
& E_{0}=\frac{N(N-1)}{2 V} \nu_{0}-\sum_{\mathbf{k} \neq 0} \frac{\hbar^{2} k^{2}}{8 m}\left(\alpha_{k}-1\right)^{2}+ \\
& +\sum_{\mathbf{k} \neq 0} \frac{\hbar^{2} k^{2}}{4 m} \alpha_{k}\left(\sqrt{1+\left(\frac{\beta_{k}}{2 \alpha_{k}}\right)^{2}}+\frac{\beta_{k}}{2 \alpha_{k}}-1\right)
\end{aligned}
$$

was calculated in [22] with the deformed Heisenberg algebra (8). When $\beta_{k} \rightarrow 0$, we obtain the groundstate energy of the Bose liquid in Bogoliubov's approximation.

The motion of the impurity can be described by the Hamiltonian $\widehat{H}_{I}$, and the wave function of the impurity is a plane wave. We now obtain a correction to the ground-state energy of the system "Bose liquid plus impurity". Suppose that the interaction between atoms of the liquid and the impurity (7) is a small perturbation. It should be noted that the probability of the transition to higher excited states that correspond to quantum numbers $n_{\mathbf{k}, \mu}>1$ from $(\mathbf{k}, \mu)$-modes is low.

The total energy of the system "Bose liquid plus impurity" in the limit of low temperature according to perturbation theory can be written as

$E_{q}=E_{q}^{(0)}+E_{q}^{(1)}+E_{q}^{(2)}$,

where the first term is the ground-state energy of the system in the zeroth approximation:

$E_{q}^{(0)}=E_{0}+\frac{\hbar^{2} q^{2}}{2 M}$.

The second term is the diagonal matrix element of the perturbation operator calculated on the ground-state wave functions of the system (when $n_{\mathbf{k}, \mu}=0$ ):

$E_{q}^{(1)}=\left\langle 0, \mathbf{q}\left|\hat{H}_{\text {int }}\right| 0, \mathbf{q}\right\rangle$.

ISSN 2071-0194. Ukr. J. Phys. 2017. Vol. 62, No. 2 
Having used the evident form of operator (7) and making replacement $\rho_{\mathbf{k}, \mu}=\widehat{Q}_{\mathbf{k}, \mu}$, we obtain

$\widehat{H}_{\text {int }}=$

$=\frac{N}{V} \bar{\nu}_{0}+\frac{\sqrt{N}}{V} \sum_{\mathbf{k}_{1} \neq 0} \bar{\nu}_{k_{1}}\left(\widehat{Q}_{\mathbf{k}_{1}, c}-i \widehat{Q}_{\mathbf{k}_{1}, s}\right) e^{i \mathbf{k}_{1} \mathbf{r}}$.

Since we consider corrections to the ground state, we obtain in the first order (because $\mathbf{k}_{1} \neq 0$ ):

$E_{q}^{(1)}=\frac{N}{V} \bar{\nu}_{0}$

The second-order correction to the ground-state energy of the system can be written as

$E_{q}^{(2)}=\sum_{\mathbf{q}^{\prime} \neq \mathbf{0}} \sum_{\mathbf{k}^{\prime} \neq \mathbf{0}} \frac{\left|\left\langle\mathbf{k}^{\prime}, \mathbf{q}^{\prime}\left|\hat{H}_{i n t}\right| 0, \mathbf{q}\right\rangle\right|^{2}}{\hbar^{2} q^{2} / 2 M-\hbar^{2} q^{\prime 2} / 2 M-E_{k^{\prime}}}$,

where $E_{k^{\prime}}$ is the spectrum of elementary excitations of liquid ${ }^{4} \mathrm{He}$, which can be represented as a function of the structure factor of the Bose liquid $S_{k^{\prime}}$. In the deformed case, we have

$E_{k^{\prime}}=\frac{\hbar^{2} k^{\prime 2}}{2 m S_{k^{\prime}}}+\frac{\hbar^{2} k^{\prime 2}}{2 m} \beta_{k^{\prime}}$.

Equation (28) shows that the deformation parameter $\beta_{k^{\prime}}$ takes negative values. The experimentally measured spectrum of elementary excitations is lower than the theoretical one calculated in the approximation of pair correlations. To find correction (27), we have to calculate the off-diagonal matrix element on the wave functions given by Eq. (9), when $n_{\mathbf{k}, \mu}=0$, and Eq. (17). Details of the calculation are presented in Appendix A. Now, the off-diagonal matrix element for the negative values of the deformation parameter can be written as follows:

$\left|\left\langle\mathbf{k}^{\prime}, \mathbf{q}^{\prime}\left|\widehat{H}_{\text {int }}\right| 0, \mathbf{q}\right\rangle\right|^{2}=$
$=\frac{N}{V^{2}} \bar{\nu}_{k^{\prime}}^{2} \frac{1}{\beta_{k^{\prime}}} \frac{(\nu-1)(\nu-1 / 2)^{2}}{\nu^{2}}\left[\frac{\Gamma(\nu-1 / 2)}{\Gamma(\nu)}\right]^{4}$,

where $\nu$ is taken from Eq. (14), when $k=k^{\prime}$. We write the second-order correction to the ground-state energy (27), by taking the off-diagonal matrix element (29) into account at $\beta_{k}<0$. Expression (27) can be expanded in a series in powers of $\mathbf{k}^{\prime}$ :

$E_{q}^{(2)}=-\frac{N}{V^{2}} \sum_{\mathbf{k}^{\prime} \neq 0} \bar{\nu}_{k^{\prime}}^{2} \frac{1}{\beta_{k^{\prime}}} \frac{(\nu-1)(\nu-1 / 2)^{2}}{\nu^{2}} \times$

ISSN 2071-0194. Ukr. J. Phys. 2017. Vol. 62, No. 2

$$
\begin{aligned}
& \times\left[\frac{\Gamma(\nu-1 / 2)}{\Gamma(\nu)}\right]^{4} \frac{1}{\hbar^{2} k^{\prime 2} / 2 M+E_{k^{\prime}}} \times \\
& \times\left[1+\left(\frac{2\left(\mathbf{k}^{\prime} \mathbf{q}\right)}{k^{\prime 2}+2 E_{k^{\prime}} M / \hbar^{2}}\right)^{2}+\ldots\right] .
\end{aligned}
$$

We have taken into account that $\mathbf{q}^{\prime}=\mathbf{q}+\mathbf{k}^{\prime}$. We also remark that the terms $\sum_{\mathbf{k}^{\prime} \neq 0}\left(\mathbf{k}^{\prime} \mathbf{q}\right)^{s}$, which appear in decomposition (30), are equal to zero. Since the lowest excited states of the system "Bose liquid plus impurity" correspond to sound waves, the expansion in powers of $\mathbf{k}^{\prime}$ is allowed for any value of the wave vector $\mathbf{k}^{\prime}$. We write the second-order correction in the limit $\beta_{k^{\prime}} \rightarrow 0$. With regard for the approximate expansion for the Gamma-function, $\Gamma(\nu+a)=$ $\sqrt{2 \pi} e^{-\nu} \nu^{\nu+a-1 / 2}$, we can easily show that the correction to the ground-state energy (30) of the system is the same as in the non-deformed case:

$E_{q}^{(2)}=$

$=-\frac{N}{V^{2}} \sum_{\mathbf{k}^{\prime} \neq \mathbf{0}} \frac{\nu_{k^{\prime}}^{2} / \alpha_{k^{\prime}}}{\hbar^{2} k^{2} / 2 M+\hbar^{2}\left(\mathbf{k}^{\prime} \mathbf{q}\right) / M+E_{k^{\prime}}}$.

\section{Effective Mass}

Let us write the second-order correction to the ground-state energy (30) of the system as

$E_{q}^{(2)}=E_{q=0}^{(2)}-$

$-\frac{N}{V^{2}} \sum_{\mathbf{k}^{\prime} \neq 0}\left(\frac{\hbar^{2} \bar{\nu}_{k^{\prime}}}{M}\right)^{2} \frac{\left(\mathbf{k}^{\prime} \mathbf{q}\right)^{2}}{\left[\hbar^{2} k^{\prime 2} / 2 M+E_{k^{\prime}}\right]^{3}} f\left(\beta_{k^{\prime}}\right)$,

where

$E_{q=0}^{(2)}=-\frac{N}{V^{2}} \sum_{\mathbf{k}^{\prime} \neq 0} \bar{\nu}_{k^{\prime}}^{2} \frac{1}{\hbar^{2} k^{\prime 2} / 2 M+E_{k^{\prime}}} f\left(\beta_{k^{\prime}}\right)$,

and the function

$f\left(\beta_{k^{\prime}}\right)=\frac{(\nu-1)(\nu-1 / 2)^{2}}{\left|\beta_{k^{\prime}}\right| \nu^{2}}\left[\frac{\Gamma(\nu-1 / 2)}{\Gamma(\nu)}\right]^{4}$.

In the limit $\beta_{k^{\prime}} \rightarrow 0$, the function $f\left(\beta_{k^{\prime}}\right) \rightarrow 1 / \alpha_{k^{\prime}}$.

The first-order corrections to the ground-state energy together with term (33) gives the immersion energy of the impurity atom in the Bose liquid:

$E_{i}=\rho \bar{\nu}_{0}-\frac{1}{N} \sum_{\mathbf{k}^{\prime} \neq 0} \frac{\left(\rho \bar{\nu}_{k^{\prime}}\right)^{2}}{\hbar^{2} k^{\prime 2} / 2 M+E_{k^{\prime}}} f\left(\beta_{k^{\prime}}\right)$,

where $\rho=N / V$ is the density of ${ }^{4} \mathrm{He}$. 
The Fourier coefficient $\bar{\nu}_{0}$ can be written as a function of the speed of sound in liquid helium. To do that, we use the well-known thermodynamic relation:

$m c^{2}=N \frac{\partial^{2} E_{0}}{\partial N^{2}}$.

We note that the speed of sound in liquid helium at $T=0 \mathrm{~K}$ is equal to $c=238.2 \mathrm{~m} / \mathrm{s}$. As a result, we obtain

$\bar{\nu}_{0}=\frac{m c^{2}}{\rho_{0}}+\frac{1}{2 N \rho_{0}} \sum_{\mathbf{k} \neq 0} \frac{\hbar^{2} k^{2}}{2 m} S_{k} \times$

$\times\left(S_{k}-\frac{1}{S_{k}}\right)^{2}\left[1-\left(\frac{\beta_{k} S_{k}}{2}\right)^{2}\right]^{3 / 2}$,

where $\rho_{0}=0.0218 \AA^{-3}$ is an equilibrium density of liquid helium. The expression for the total energy of the system "Bose liquid plus impurity" can be written as follows:

$E_{q}=E_{0}+E_{i}+\frac{\hbar^{2} q^{2}}{2 M^{*}}$,

where $M^{*}$ is the effective mass of the impurity:

$$
\begin{aligned}
& \frac{M}{M^{*}}=1-\frac{N}{V^{2}} \sum_{\mathbf{k}^{\prime} \neq 0} \bar{\nu}_{k^{\prime}}^{2}\left(\frac{2 M}{\hbar^{2}}\right)^{2} \times \\
& \times \frac{4\left(\mathbf{k}^{\prime} \mathbf{q}\right)^{2} f\left(\beta_{k^{\prime}}\right)}{\left[k^{\prime 2}+2 E_{k^{\prime}} M / \hbar^{2}\right]^{3}} .
\end{aligned}
$$

For the numerical evaluation of the effective mass of the impurity, we will model the deformation parameter by a function that depends on the structure factor. The structure factor can be taken from the extrapolated data on the structure factor for the Bose liquid at $T=0 \mathrm{~K}$ :

$\beta_{k^{\prime}}=-S_{k^{\prime}}\left|S_{k^{\prime}}-1\right|^{3}$.

We note that the choice of the deformation parameter gives the correct behavior in the long-wavelength domain. Function (40) can be used for finding the physical quantities in the limit of $T \rightarrow 0$. We can suggest other model of the function, which would reproduce the corrections to physical characteristics of the system "Bose liquid plus impurity" in the post-RPA approximation.

Now, we calculate the effective mass of the impurity atom ${ }^{3} \mathrm{He}$, by supposing that the deformation parameter takes the form (40). We write expression (39) for the effective mass in the thermodynamical limit $(N \rightarrow \infty, V \rightarrow \infty, N / V=$ const $)$. The sum over the wave vector $\mathbf{k}^{\prime}$ in that relation can be replaced by an integral. Having performed the integration over angular variables, we arrive at the expression

$$
\begin{aligned}
& \frac{M}{M^{*}}=1-\frac{2}{3 \pi^{2}} \rho \int_{0}^{\infty} k^{\prime 4} \times \\
& \times \frac{\bar{\nu}_{k^{\prime}}^{2}\left(2 M / \hbar^{2}\right)^{2}}{\left[k^{\prime 2}+2 E_{k^{\prime}} M / \hbar^{2}\right]^{3}} f\left(\beta_{k^{\prime}}\right) \mathrm{d} k^{\prime} .
\end{aligned}
$$

The Fourier image of the interaction potential between the Bose particles and the impurity atom $\bar{\nu}_{k^{\prime}}$ can be expressed as a function of the structure factor of the Bose liquid. Let us suppose that $\nu_{k^{\prime}}=\bar{\nu}_{k^{\prime}}$. Then, for the ${ }^{3} \mathrm{He}$ impurity, we can write

$\bar{\nu}_{k^{\prime}}=\frac{\hbar^{2} k^{\prime 2}}{4 m \rho_{0}}\left(\frac{1}{S_{k^{\prime}}^{2}}-1\right)$.

This assumption is also correct for the elements, for which the outer $s$-electron shells are filled completely, in particular, this fact holds for the inert gases. We note that the equality $\nu_{k^{\prime}}=\bar{\nu}_{k^{\prime}}$ does not take place for other kinds of impurities, because the Fourier transforms exist just for few types of the interaction potential. For the equilibrium density of the liquid helium $\left(\rho_{0}=0.0218 \AA^{-3}\right)$, the effective mass of the atom ${ }^{3} \mathrm{He}$ is equal to $M^{*}=1.78 M$.

An important characteristic of the system "Bose liquid plus impurity" is the separation energy. The separation energy is the energy that is necessary for the removal of an impurity atom from the mixture ${ }^{3} \mathrm{He}-{ }^{4} \mathrm{He}$ (when the concentration of impurities is very low). The value of separation energy for a single impurity atom ${ }^{3} \mathrm{He}$ (when the density of helium is $\rho_{0}=0.0218 \AA^{-3}$ ) is equal to $B^{\exp }=2.76 \mathrm{~K}[11$.

We now calculate the separation energy $B$ with the deformation parameter (40) for different values of the ${ }^{4} \mathrm{He}$ density. To do that, we replace the impurity atom with the atom of the Bose liquid. Then the groundstate energy $(22)$ of the system of $(N+1)$ Bose particles is decomposed into a series in powers of $N$. We obtain the expression

$\Delta E=E_{i}-\left(\frac{\partial E_{0}}{\partial N}\right)_{T}$.

It is the so-called substitution energy of the system "Bose liquid plus impurity atom" provided that the 
Fourier image of the impurity-liquid interaction potential equals the Fourier image of the liquid-liquid potential. The explicit form of Eq. (43) is

$$
\begin{aligned}
& \Delta E=\frac{1}{4 N} \sum_{\mathbf{k} \neq 0} \frac{\hbar^{2} k^{2}}{2 m}\left(\alpha_{k}^{2}-1\right)\left(2-\frac{1}{\alpha_{k}}-\right. \\
& \left.-\frac{1}{\sqrt{1+\left(\beta_{k} / 2 \alpha_{k}\right)^{2}}}-\left(\frac{\rho}{\rho_{0}}\right)^{2} \frac{\left(\alpha_{k}^{2}-1\right) f\left(\beta_{k}\right)}{m / M+\alpha_{k}+\beta_{k}}\right),
\end{aligned}
$$

where the Bogoliubov factor $\alpha_{k}$ depends on the deformation parameter:

$\alpha_{k}^{2}=\frac{1}{S_{k}^{2}}-\frac{\beta_{k}^{2}}{4}$.

In the limit $\beta_{k} \rightarrow 0$ and with $\rho_{0}$, the substitution energy given by Eq. (44) reads

$\Delta E=-\frac{1}{4 N}\left(1-\frac{m}{M}\right) \sum_{\mathbf{k} \neq 0} \frac{\hbar^{2} k^{2}}{2 m} \frac{\left(\alpha_{k}-1\right)^{2}}{\alpha_{k}} \frac{1+\alpha_{k}}{m / M+\alpha_{k}}$.

When $m=M$, we obtain $\Delta E=0$.

The definition of the separation energy from [11] can be written, in our notation, as

$-B=\Delta E+\frac{E_{0}}{N}$.

Here, the ground-state energy of the Bose liquid $E_{0} / N$ is given by Eq. (21) and can be presented for one particle as [22]

$\frac{E_{0}}{N}=\frac{m c^{2}}{2} \frac{\rho}{\rho_{0}}+\frac{1}{32 \pi^{2}} I_{1}+\frac{1}{8 \pi^{2}} I_{2}$,

where

$I_{1}=\frac{\hbar^{2}}{2 m \rho_{0}} \int_{0}^{\infty} k^{4}\left[\frac{\left(1-S_{k}^{2}\right)^{2}}{S_{k}}+\left(2\left(S_{k}^{2}-1\right)+\right.\right.$

$\left.\left.+\left(\frac{\beta_{k} S_{k}}{2}\right)^{2}\right)\left(\frac{\beta_{k} S_{k}}{2}\right)^{2}\right] \mathrm{d} k$

$I_{2}=\frac{\hbar^{2}}{2 m \rho} \int_{0}^{\infty} k^{4}\left[\beta_{k}+\frac{\beta_{k}^{2}}{4}-\left(1-\frac{1}{S_{k}}\right)^{2}\right] \mathrm{d} k$.

We write Eqs. (49) and (50) similarly in the thermodynamic limit, by considering $\sum_{\mathbf{k} \neq 0} \rightarrow$ $\rightarrow 1 /\left(8 \pi^{3} \rho\right) \int \mathrm{d} \mathbf{k}$.

To evaluate the separation energy (47) for densities that are not equal to the equilibrium density $\rho_{0}$, the structure factor $S_{k}$ in Eqs. (44), (49), and (50) should be renormalized. To perform this procedure, we rewrite the structure factor in the following form:

$\frac{1}{S_{k}^{2}}=1+\frac{\rho}{\rho_{0}}\left(\frac{1}{S_{\exp }^{2}(k)}-1\right)$

where $S_{\exp }(k)$ is the structure factor of the Bose liquid taken from experimental measurements.

Table 2 shows the values of separation energy that we have calculated by formula (47) in the deformed case. For comparison, the second and third columns represent the separation energies for the same densities that obtained in work [11]. We remark that, in the calculation of the separation energy, the Brillouin-Wigner perturbation theory was used. Here, the separation energy $B$ for the system of $(N-1)$ Bose particles and a single impurity atom is given. We also present the results of the separation energy from work [24]. In our work, the immersion energy is defined as the difference of the energy of the system "Bose liquid plus impurity," which is calculated by the variational method in the approximation of pair correlations, and the energy of the pure Bose liquid. The effective mass for different values of the density of the Bose liquid is shown in Table 1.

We note that one can propose other model functions for the deformation parameter provided that the contributions to physical characteristics of the system

Table 1. Effective mass of the impurity

atom ${ }^{3} \mathrm{He}$ for different values of the density of ${ }^{4} \mathrm{He}$

\begin{tabular}{|c|c|c|c|c|c|}
\hline $\begin{array}{c}\rho, \\
\AA^{-3}\end{array}$ & $\begin{array}{c}M^{*} / M \\
\text { (this work) }\end{array}$ & $\begin{array}{c}M^{*} / M \\
\left.\text { (if } \beta_{k}=0\right)\end{array}$ & $\begin{array}{c}M^{*} / M, \\
{[19]}\end{array}$ & $\begin{array}{c}M^{*} / M, \\
{[17]}\end{array}$ & $\begin{array}{c}M^{*} / M, \\
{[23]}\end{array}$ \\
\hline 0.0218 & 1.78 & 1.71 & 2.15 & 2.20 & 2.09 \\
0.0240 & 2.13 & 2.00 & 2.39 & 2.36 & 2.34 \\
0.0254 & 2.50 & 2.27 & 2.62 & 2.72 & 2.55 \\
\hline
\end{tabular}

Table 2. Separation energy of the impurity atom ${ }^{3} \mathrm{He}$ for different values of the density of ${ }^{4} \mathrm{He}$

\begin{tabular}{|c|c|c|c|c|}
\hline $\begin{array}{c}\rho, \\
\AA^{-3}\end{array}$ & $\begin{array}{c}B, \mathrm{~K} \\
\text { (this work) }\end{array}$ & $\begin{array}{c}B, \mathrm{~K} \\
\left.\text { (if } \beta_{k}=0\right)\end{array}$ & $\begin{array}{c}B, \mathrm{~K}, \\
{[11]}\end{array}$ & $\begin{array}{c}B, \mathrm{~K}, \\
{[24]}\end{array}$ \\
\hline 0.0218 & 4.56 & 2.09 & 1.39 & 2.02 \\
0.0226 & 4.09 & 1.66 & 1.15 & 2.05 \\
0.0234 & 3.58 & 1.21 & 0.88 & 2.09 \\
0.0242 & 3.05 & 0.74 & 0.51 & 2.09 \\
0.0250 & 2.53 & 0.27 & 0.13 & 2.05 \\
0.0258 & 2.00 & -0.16 & -0.36 & 1.98 \\
\hline
\end{tabular}


"Bose liquid plus impurity" will reproduce the results in the post-RPA approximation. For the sake of comparison of our results, we choose the deformation parameter in the form

$\beta_{k}=-\beta_{0} e^{-\left(1-k / k_{0}\right)^{2}}$.

As $k \rightarrow \infty$, we can set $\beta_{k}=-\beta_{0}$. We choose the coefficient $\beta_{0}$ so that the theoretical excitation spectrum (28) reproduces the experimental one. The wave vector in this case takes the value $k_{0}=0.98 \AA^{-1}$. This choice of the coefficient $k_{0}$ and $\beta_{0}$ provides such a value of the ground-state energy (48) that is consistent with the experimental data for the ground-state energy of the Bose liquid.

\section{APPENDIX A}

We write the off-diagonal matrix elements in the form

$$
\begin{aligned}
& \left\langle\mathbf{k}^{\prime}, \mathbf{q}^{\prime}\left|\widehat{H}_{\mathrm{int}}\right| 0, \mathbf{q}\right\rangle=\delta_{\mathbf{k}_{\mathbf{1}}, \mathbf{q}^{\prime}-\mathbf{q}} \frac{\sqrt{N}}{V} \bar{\nu}_{k_{1}} \times \\
& \times\left(\left\langle\mathbf{k}^{\prime}\left|\widehat{Q}_{\mathbf{k}_{\mathbf{1}}, c}\right| 0\right\rangle-i\left\langle\mathbf{k}^{\prime}\left|\widehat{Q}_{\mathbf{k}_{\mathbf{1}}, s}\right| 0\right\rangle\right) .
\end{aligned}
$$

We calculate one of the terms in brackets, by using the following expression for the wave function of the first excited state of a Bose liquid (18):

$$
\begin{aligned}
& \left\langle\mathbf{k}^{\prime}\left|\widehat{Q}_{\mathbf{p}, \mu^{\prime}}\right| 0\right\rangle= \\
& =\sqrt{2(\nu-1)}\left\langle 0\left|\sinh \left(q_{\mathbf{k}^{\prime}, \mu} \sqrt{\left|\beta_{k^{\prime}}\right|}\right) \widehat{Q}_{\mathbf{p}, \mu^{\prime}}\right| 0\right\rangle .
\end{aligned}
$$

Here, we have introduced the notation $\mathbf{p}=\mathbf{q}^{\prime}-\mathbf{q}$ and $\mu^{\prime}=c$ or $\mu^{\prime}=s$. Equation (54) is not equal to zero, when $\mathbf{p}=\mathbf{k}^{\prime}$ and $\mu^{\prime}=\mu$. With regard for the explicit form of the ground-state wave function, the matrix element can be written as follows:

$$
\begin{aligned}
& \left\langle\mathbf{k}^{\prime}\left|\widehat{Q}_{\mathbf{p}, \mu^{\prime}}\right| 0\right\rangle=\frac{1}{\sqrt{\left|\beta_{k^{\prime}}\right|}} \sqrt{2(\nu-1)} \frac{\Gamma(\nu+1 / 2)}{\Gamma(1 / 2) \Gamma(\nu)} \times \\
& \times \int_{-\infty}^{\infty} \frac{\sinh ^{2}\left(q_{\mathbf{k}^{\prime}, \mu} \sqrt{\left|\beta_{k^{\prime}}\right|}\right)}{\cosh ^{2 \nu+1}\left(q_{\mathbf{k}^{\prime}, \mu} \sqrt{\left|\beta_{k^{\prime}}\right|}\right)} \mathrm{d} q_{\mathbf{k}^{\prime}, \mu} .
\end{aligned}
$$

The last integral can be reduced to the Beta-function. Finally, we obtain

$$
\begin{aligned}
& \left\langle\mathbf{k}^{\prime}, \mathbf{q}^{\prime}\left|\widehat{H}_{\mathrm{int}}\right| 0, \mathbf{q}\right\rangle= \\
& =\frac{\sqrt{N}}{V} \bar{\nu}_{k^{\prime}}(1-i) \sqrt{\frac{\nu-1}{2\left|\beta_{k^{\prime}}\right|}} \frac{\nu-1 / 2}{\nu}\left[\frac{\Gamma(\nu-1 / 2)}{\Gamma(\nu)}\right]^{2} .
\end{aligned}
$$

The authors appreciate the help of Dr. Mykola Stetsko in the preparation of this article.

1. M. Schecter, D.M. Gangardt, A. Kamendev. Dynamics and Bloch oscillations of mobile impurities in onedimensional quantum liquids. Ann. Phys. 327, 639 (2012) [DOI: 10.1016/j.aop.2011.10.001].
2. M. Rinek, C. Bruder. Effects of a single fermion in a BoseJosephson junction. Phys. Rev. A 83, 023608 (2011) [DOI: 10.1103/PhysRevA.83.023608].

3. S. Schmid, A. Härter, J.H. Denschlag. Dynamics of a cold trapped ion in a Bose-Einstein condensate. Phys. Rev. Lett. 105, 133202 (2010) [DOI: 10.1103/PhysRevLett.105.133202].

4. W. Casteels, J. Tempere, J.T. Devreese. Bipolarons and multipolarons consisting of impurity atoms in a Bose-Einstein condensate. Phys. Rev. A 88, 013613 (2013) [DOI: 10.1103/PhysRevA.88.013613].

5. Y.E. Shchadilova, F. Grusdt, A. Rubtsov, E. Demler. Polaronic mass renormalization of impurities in Bose-Einstein condensates: Correlated Gaussian-wave-function approach. Phys. Rev. A 93, 043606 (2016) [DOI: 10.1103/ PhysRevA.93.043606].

6. R.S. Christensen, J. Levinsen, G. Bruun. Quasiparticle properties of a mobile impurity in a Bose-Einstein condensate. Phys. Rev. Lett. 115, 160401 (2015) [DOI: 10.1103/ PhysRevLett.115.160401].

7. J.N. Fuch, D.M. Gangardt, T. Keilmann. Spin waves in a one-dimensional spinor Bose gas. Phys. Rev. Lett. 95 150402 (2015) [DOI: 10.1103/PhysRevLett.95.150402].

8. M.W. Reynolds, M.E. Hayden, W.N. Hardy. Hyperfine resonance of atomic deuterium at 1 K. J. Low. Temp. Phys. 84, 87 (1991) [DOI: 10.1007/BF00681618].

9. L.A. Peña, S. Giorgini. Impurity in a Bose-Einstein condensate: Study of the attractive and repulsive branch using quantum Monte Carlo methods. Phys. Rev. A 92, 033612 (2015) [DOI: 10.1103/PhysRevA.92.033612].

10. V.A. Slusarev, M.A. Strzemechny. On the theory of the energy spectrum of $\mathrm{He}^{3}$ diluted mixtures in superfluid $\mathrm{He}^{4}$. Ukr. J. Phys. 14, 450 (1969) (in Russian).

11. T.B. Davison, E. Finberg. Theory of a $\mathrm{He}^{3}$ atom in liquid $\mathrm{He}^{4}$ at $T=0$. Phys. Rev. 178, 306 (1969) [DOI: 10.1103/PhysRev.178.306].

12. W.E. Massey, C.W. Woo. Variational calculations on liquid $\mathrm{He}^{4}$ and $\mathrm{He}^{3}$. Phys. Rev. 164, 256 (1967) [DOI: 10.1103/PhysRev.164.256].

13. J.C. Owen. Effective mass of one ${ }^{3} \mathrm{He}$ atom in liquid ${ }^{4}$ He. Phys. Rev. B 23, 5815 (1981) [DOI: 10.1103/PhysRevB.23.5815].

14. I.O. Vakarchuk, V.V. Babin. Impurity effective mass in superfluid ${ }^{4}$ He. Condens. Matter Phys. 1, 161 (1998) [DOI: 10.5488/CMP.1.1.161].

15. E.C. Svensson, V.F. Sears, A.D. Woods, P. Martel. Neutron-diffraction study of the static structure factor and pair correlations in liquid ${ }^{4} \mathrm{He}$. Phys. Rev. B 21, 3638 (1980) [DOI: 10.1103/PhysRevB.21.3638].

16. I.O. Vakarchuk, V.V. Babin. Low-temperature asymptotic behaviour of the impurity effective mass in superfluid ${ }^{4} \mathrm{He}$. J. Phys. Stud. 3, 468 (1999) (in Ukrainian).

17. J. Boronat, J. Casulleras. Quantum Monte Carlo study of static properties of one ${ }^{3} \mathrm{He}$ atom in superfluid ${ }^{4} \mathrm{He}$. Phys. Rev. B 59, 8844 (1999) [DOI: 10.1103/PhysRevB.59.8844].

ISSN 2071-0194. Ukr. J. Phys. 2017. Vol. 62, No. 2 
18. E.A. Pashitskii. The role of pair correlations in the formation of the ground state and the elementary excitation spectrum in a superfluid Bose liquid (A review). Low. Temp. Phys. 25, 81 (1999) [DOI: 10.1063/1.593709].

19. R. Simmons, R.M. Mueller. Specific heat of ${ }^{3} \mathrm{He} /{ }^{4} \mathrm{He}$ mixtures at low temperatures and high ${ }^{3} \mathrm{He}$ concentrations. J. Phys. Suppl. 46, 201 (1996) [DOI: 10.1007/ BF02569516].

20. S. Will, T. Best, S. Braum. Coherent interaction of a single fermion with a small bosonic field. Phys. Rev. Lett. 106, 115305 (2011) [DOI: 10.1103/PhysRevLett.106.115305].

21. A.J. Kempf. Uncertainty relation in quantum mechanics with quantum group symmetry. J. Math. Phys. 35, 4483 (1994) [DOI: 10.1063/1.530798].

22. I.O. Vakarchuk, G.I. Panochko. The theory of a manyboson system with the deformed Heisenberg algebra. Condens. Matter Phys. 3, 33002 (2015) [DOI: 10.5488/ CMP.18.33002].

23. E. Krotscheck, M. Saarela, K. Schorkhuber, R. Zillich. Concentration dependence of the effective mass of ${ }^{3} \mathrm{He}$ atoms in ${ }^{3} \mathrm{He}^{-}{ }^{4} \mathrm{He}$ mixtures. Phys. Rev. Lett. 80, 4709 (1998) [DOI: 10.1103/PhysRevLett.80.4709].
24. I.O. Vakarchuk, G.I. Panochko. Impurity states in a manyboson system. J. Phys. Stud. 16, 4601 (2012).

Received 14.09.16

I.О. Вакарчук, Г. Паночко

ЕФЕКТИВНА МАСА ДОМШШКОВОГО

АТОМА В БОЗЕ-РІДИНІ 3 ДЕФОРМОВАНОЮ

АЛГЕБРОЮ ГАЙЗЕНБЕРГА

$\mathrm{P}$ е $з$ ю м е

В роботі розглянуто рух домішки ${ }^{3} \mathrm{He}$ в бозе-рідині. Особливістю використаного нами підходу є врахування багаточастинкових кореляцій між атомами рідини деформованою алгеброю Гайзенберга. В ролі узагальнених координат вибрано колективні змінні, що є коефіцієнтами Фур'є флуктуації густини бозе-частинок. Хвильову функцію системи в нульовому наближенні описуємо добутком хвильової функції рідкого гелію-4 на мові деформованих комутаційних співвідношень між узагальненими координатами і спряженими імпульсами та плоскою хвилею домішкового атома. Вважаючи бозон-домішкову взаємодію малим збуренням, знайдено поправки до енергії основного стану системи “бозе-рідина плюс домішка", а також обчислено ефективну масу домішкового атома ${ }^{3} \mathrm{He}$. 